\title{
Automated Fetal Brain Segmentation from 2D MRI Slices for Motion Correction
}

\author{
K. Keraudren ${ }^{\mathrm{a}, *}$, M. Kuklisova-Murgasova ${ }^{\mathrm{b}}$, V. Kyriakopoulou ${ }^{\mathrm{b}}$, C. Malamateniou ${ }^{\mathrm{b}}$, M.A. Rutherford ${ }^{\mathrm{b}}$, B. Kainz $^{\mathrm{a}}$, J.V. Hajnal $^{\mathrm{b}}$, \\ D. Rueckert ${ }^{\mathrm{a}}$ \\ ${ }^{a}$ Biomedical Image Analysis Group, Department of Computing, Imperial College London, SW7 2AZ, UK \\ ${ }^{b}$ Centre for the Developing Brain, Division of Imaging Sciences $\mathcal{E}$ Biomedical Engineering, King's College London, St. Thomas' Hospital, SE1 7EH, UK
}

\begin{abstract}
Motion correction is a key element for imaging the fetal brain in-utero using Magnetic Resonance Imaging (MRI). Maternal breathing can introduce motion, but a larger effect is frequently due to fetal movement within the womb. Consequently, imaging is frequently performed slice-by-slice using single shot techniques, which are then combined into volumetric images using slice-tovolume reconstruction methods (SVR). For successful SVR, a key preprocessing step is to isolate fetal brain tissues from maternal anatomy before correcting for the motion of the fetal head. This has hitherto been a manual or semi-automatic procedure. We propose an automatic method to localize and segment the brain of the fetus when the image data is acquired as stacks of 2D slices with anatomy misaligned due to fetal motion. We combine this segmentation process with a robust motion correction method, enabling the segmentation to be refined as the reconstruction proceeds. The fetal brain localization process uses Maximally Stable Extremal Regions (MSER), which are classified using a Bag-of-Words model with Scale-Invariant Feature Transform (SIFT) features. The segmentation process is a patch-based propagation of the MSER regions selected during detection, combined with a Conditional Random Field (CRF). The gestational age (GA) is used to incorporate prior knowledge about the size and volume of the fetal brain into the detection and segmentation process. The method was tested in a ten-fold cross-validation experiment on 66 datasets of healthy fetuses whose GA ranged from 22 to 39 weeks. In $85 \%$ of the tested cases, our proposed method produced a motion corrected volume of a relevant quality for clinical diagnosis, thus removing the need for manually delineating the contours of the brain before motion correction. Our method automatically generated as a side-product a segmentation of the reconstructed fetal brain with a mean Dice score of $93 \%$, which can be used for further processing.
\end{abstract}

Keywords:

Fetal MRI, Motion correction, Brain extraction, MSER, Bag-of-Words, SIFT

\section{Introduction}

Magnetic Resonance Imaging (MRI) is a primary tool for clinical investigation of the brain and for neuroscience. High resolution imaging with volumetric coverage using stacks of slices or true three dimensional (3D) methods is widely available and provides rich data for image analysis. However such detailed volumetric data generally takes several minutes to acquire and requires the subject to remain still or move only small distances during acquisition. Fetal brain imaging introduces a number of additional challenges (Malamateniou et al., 2013). Maternal breathing may move the fetus and the fetus itself can and does spontaneously move during imaging. These movements are unpredictable and may be large, particularly involving substantial head rotations. As a result, most fetal imaging is performed using single shot methods to acquire two dimensional (2D) slices, such as single shot Fast Spin Echo, ssFSE, (Jiang et al., 2007) and Echo-Planar Imaging, EPI, (Chen and Levine, 2001). These methods can freeze fetal motion, so that each individual slice is generally artifact free, but stacks of

\footnotetext{
${ }^{*}$ Corresponding author

Email address: kevin.keraudren10@imperial.ac.uk (K. Keraudren)
}

slices required to provide whole brain coverage may be mutually inconsistent.

Motion correction methods have revolutionized MRI of the fetal brain by reconstructing a high-resolution 3D volume of the fetal brain from such motion corrupted stacks of 2D slices. Such reconstructions are valuable for both clinical and research applications. They enable a qualitative assessment of abnormalities suspected on antenatal ultrasound scans (Rutherford et al., 2008) and are an essential tool to gain a better understanding of the development of the fetal brain, pushing back the frontiers of neuroscience (Studholme and Rousseau, 2013).

However, motion correction and reconstruction methods still require a substantial amount of manual data pre-processing. This makes direct clinical application of the methods impractical and is excessively time consuming in large cohort research studies.

Therefore, we propose a fully automated tightly coupled segmentation and reconstruction approach and present the following contributions in this paper:

- We develop an automated method to mask the fetal brain in every 2D slice of every 3D stack and designed it to serve as a preprocessing step for motion correction (Section 3.2). We thus seek an optimal segmentation that tackles both the 
motion between different stacks as well as the motion between the interleaved packets of a single stack. This is in contrast with a manual initialization of the motion correction procedure which typically propagates one manual mask to the remaining stacks after rigid volumetric registration.

- We propose the application of a patch-based Random Forest classifier to obtain a probabilistic slice-by-slice segmentation of the brain, which is then refined with a 3D Conditional Random Field (Section 3.2). The novelty of this approach is to perform online learning with a global classifier, whereas a typical patch-based segmentation uses offline learning with a local classifier. We train our Random Forest classifier using the partial segmentation resulting from the localization process described in Section 3.1.

- Given an approximate age of the fetus (gestational age), we incorporate prior knowledge of the size and volume of the fetal brain in both the detection (Section 3.1) and segmentation processes (Section 3.2). In particular, we use an estimation of the volume of the brain in order to improve the probabilistic output of the Random Forest classifier.

- During motion correction, we take advantage of the alignment of all 2D slices to produce a 3D probabilistic segmentation of the brain from all the slice-by-slice probabilistic segmentations (Section 3.3). We use again a 3D Conditional Random Field to update the segmentation of the fetal brain, thus improving the reconstruction of a highresolution volume.

The outline of this paper is as follows. In Section 2, we give an overview of related work for processing brain MRI. We closely review in Section 3.1 our approach to localize the fetal brain, first introduced in Keraudren et al. (2013), before showing in Section 3.2 how to refine this localization from a bounding box detection to a segmentation for all 2D slices of each 3D stack. In Section 3.3, we integrate this automated masking with the motion correction procedure, evolving the mask of the segmented brain during reconstruction. The resulting method is then evaluated in two steps (Section 5), first assessing the quality of the slice-by-slice segmentation, then comparing the result of motion correction using a manual and an automated initialization.

\section{Related Work}

\subsection{Processing of conventional cranial MRI}

Numerous methods have been proposed for an automated extraction of the adult brain from MRI data, a problem also known as skull stripping. The task is then to delineate the whole brain in a motion free 3D volume. Most of the proposed methods take advantage of the fact that the adult head is surrounded by air and that the brain is thus the main structure in the image. Brain extraction can then be performed using a region growing process, such as in FSL's Brain Extraction Tool (Smith, 2002) and FreeSurfer's Hybrid Watershed Algorithm (Segonne et al., 2004), or using morphological operations and edge detection such as in the Brain Surface Extractor of Shattuck et al. (2001). A more general approach is to register the image to segment with a brain template and use statistical models of brain tissues (Warfield et al., 1998). Instead of a template, the new image can also be registered to a set of already segmented images, called an atlas (Van Leemput et al., 1999). Tissue classification can then be performed, for instance by seeking similar patches from the atlas in a local neighborhood and transferring their labels to the new image, such as in BEaST (Eskildsen et al., 2012). Such a patch-based segmentation has been applied to fetal data after motion correction (Wright et al., 2012).

\subsection{Brain localization in fetal MRI}

Current methods to segment motion-free, high resolution adult brain volumes cannot be straight-forwardly applied to the extraction of the fetal brain from the unprocessed MR data. Anquez et al. (2009) first proposed a method for extracting the fetal brain from MRI with a low through-plane resolution, but their proposed pipeline, which relies on detecting the eyes and selecting a sagittal plane, is not robust to fetal motion. Taleb et al. (2013) also proposed a method based on template matching, but using templates from an age-dependent atlas of the fetal brain. This method first defines a region of interest (ROI) using the intersection of all scans and then registers this ROI to an age dependent fetal brain template, before refining the segmentation with a fusion step. Ison et al. (2012) and Kainz et al. (2014) address the variability of fetal MRI through the use of Machine Learning. Both methods rely on 3D features, either 3D Haar-like features or dense rotation invariant features, which are justified in cases of little motion, especially considering that the maternal anatomy is not moving and occupies most of the image. In Keraudren et al. (2013), we proposed a method to automatically select a bounding box around the fetal brain. This method is robust to fetal motion as it first detects the fetal brain in every $2 \mathrm{D}$ slice before aggregating the results in $3 \mathrm{D}$. In the present paper, we propose to leverage the result of the localization process to obtain a segmentation of the fetal brain in the unprocessed data instead of a box detection.

\subsection{Motion correction of the fetal head}

Several methods have been proposed to correct fetal motion and to reconstruct a single high-resolution 3D volume from orthogonal stacks of misaligned 2D slices. The most common approach is slice-to-volume registration, where slices are iteratively registered to an estimation of the reconstructed 3D volume (Rousseau et al., 2006; Jiang et al., 2007; Gholipour et al., 2010; Kuklisova-Murgasova et al., 2012). Another approach proposed by Kim et al. (2010) formulates the motion correction problem as the optimization of the intersections of all slice pairs from all orthogonally planned scans. A comprehensive overview of these methods is given in Studholme and Rousseau (2013).

In all proposed methods, the fetal head is considered a rigid object moving independently from the surrounding maternal tissues. Therefore, a manual initialization is required to isolate the fetal brain from the rest of the image. In both Rousseau 


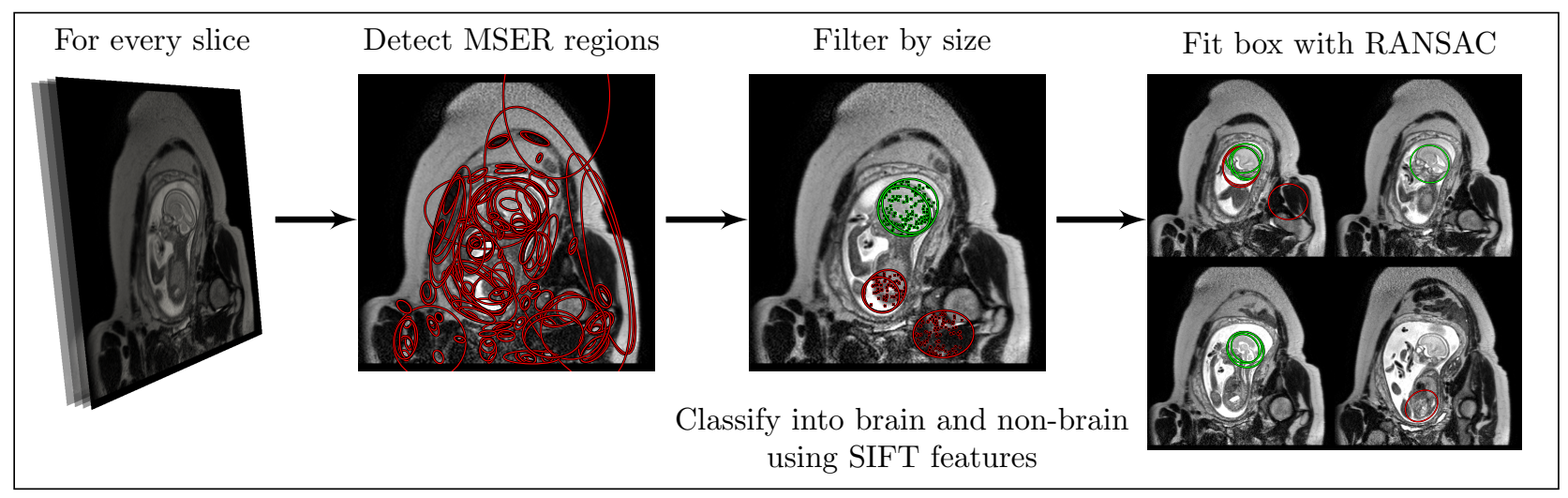

Figure 1: Pipeline to localize the fetal brain. Slice-by-slice, MSER regions are first detected and approximated by ellipses. They are then filtered by size before being classified using histograms of 2D SIFT features. Finally, a 3D bounding box is fitted to the selected MSER regions with a RANSAC procedure. In the last two steps, selected regions are shown in green while rejected regions are shown in red.

et al. (2006) and Gholipour et al. (2010), a tight bounding box is manually cropped for each stack of 2D slices. In Jiang et al. (2007) and Kuklisova-Murgasova et al. (2012), the region containing the fetal head is manually segmented in one stack, and the segmentation is propagated to the other stacks after volumeto-volume registration. Down-sampling the target volume followed by up-sampling the mask can be performed to save time during the manual segmentation. In Kim et al. (2010), a box around the brain is manually cropped in one stack and propagated to the other stacks after volume-to-volume registration. An ellipsoidal mask, which evolves while the slices are aligned is then used throughout the motion correction procedure.

Cropping only a box around the fetal brain is relatively fast but may not be enough for the reconstruction to succeed, as tissues outside the head of the fetus may mislead the registration. However, manual segmentation requires time, about 10-20min per stack of 2D slices, which can be a limitation for large scale studies. Moreover, the assumption that a mask from one stack can be propagated to all other stacks might not hold when the fetus has moved a lot between the acquisitions of each stack. Besides, slices are acquired one at a time and in an interleaved manner in order to reduce the scan time while avoiding slice cross-talk artifacts. In cases of extreme motion, the misalignment between slices can cause a 3D mask to encompass too much maternal tissue, thus requiring a slice-by-slice $2 \mathrm{D}$ segmentation. In this paper, we propose to automatically segment the fetal brain from the unprocessed data for the purpose of motion correction. The main challenges are that the fetus is in an unknown position, surrounded by maternal tissues, and may have moved between the acquisitions of 2D slices.

\section{Method}

\subsection{Bundled SIFT features brain localization pipeline}

The pipeline for detecting the fetal brain from stacks of T2 weighted single shot images, outlined in Figure 1, is a sliceby-slice approach combining state-of-the-art computer vision methods. As the data is acquired as stacks of $2 \mathrm{D}$ slices misaligned due to fetal motion, the brain is first detected independently in every 2D slice before aggregating the results in $3 \mathrm{D}$. Besides the presence of motion, the main challenge is the large variability of the appearance of the fetal brain in the $2 \mathrm{D}$ slices due to the unknown orientation of the fetus as well as anatomical changes taking place during fetal development. In order to address this challenge, the detection pipeline is based on a Bagof-Words model (Csurka et al., 2004), which is a classification approach robust to large intra-class variability. SIFT features (Scale-Invariant Feature Transform, Lowe, 1999) are used to build a visual vocabulary, enabling the representation of image regions as histograms of words. This is analogous with building histograms of words to classify text documents. The rotation invariance of SIFT features helps accommodate the unknown orientation of the fetal brain, while their scale invariance attenuates the variations due to gestational age. In order to constrain the search by incorporating prior knowledge about the expected size of the fetal brain from the gestational age, SIFT features are grouped within Maximally Stable Extremal Regions (MSER, Matas et al., 2004), which can be filtered by size, resulting in bundled SIFT features (Wu et al., 2009). The resulting Bag-ofWords classifier is trained offline on training images manually annotated with bounding boxes around the fetal brain.

MSER regions are characterized by homogeneous intensity distributions and high intensity differences at their boundary. They are connected components of the level sets of a grayscale image whose boundaries are relatively stable over a small range of intensity thresholds. They can be computed in quasi-linear time by forming the component tree of the image (Najman et al., 2004), a tree structure highlighting the inclusion relation of the connected components of the level sets in the image. The potential use of MSER regions to characterize the brain in MRI was first proposed by Donoser and Bischof (2006) who used volumetric MSER to segment simulated brain MR images. As the brain and cerebrospinal fluid appear much brighter than the surrounding bone and skin tissues in fetal T2 MRI, MSER re- 


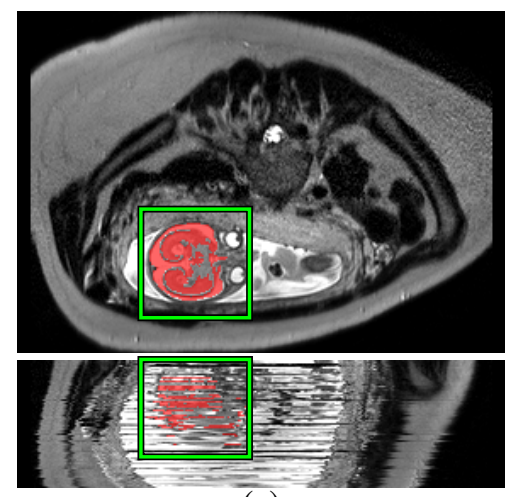

(a)

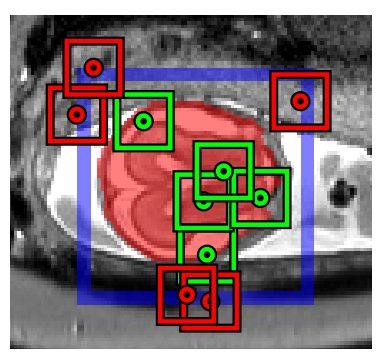

(b)

Figure 2: (a) Detected brain (green box) and selected MSER regions (red overlay). The top image shows a $2 \mathrm{D}$ slice while the bottom image is an orthogonal cut through the stack of 2D slices. (b) A Random Forest classifier is trained on patches extracted in the selected MSER regions (green boxes selected within the red overlay) and on patches extracted in a narrow band at the boundary of the detected bounding box (red boxes selected within the blue overlay).

gions are well suited to localize the brain. In order to define the regions in which SIFT features will be computed, ellipses are fitted to the MSER regions with a least-squares minimization. As the brain is well approximated by an ellipsoid, this has the advantage of recovering the shape of the brain even if the detected MSER region only corresponds to a part of the brain. Knowing the gestational age, those ellipses can be filtered by size using estimates of the occipitofrontal diameter, $O F D$, and the biparietal diameter, BPD, from 2D ultrasound studies (Snijders and Nicolaides, 2003).

Following a typical Bag-of-Words model, SIFT features are extracted from the training data and clustered using a k-means algorithm to form a vocabulary from the cluster centers. Each bundle of SIFT features can then be summarized as a histogram of words by matching each SIFT feature to its nearest neighbor in the vocabulary using the Euclidean distance. The classification task is then performed using a linear kernel Support Vector Machine (SVM) after normalization of the histograms with the $L_{2}$ norm. The SVM is trained using the bounding boxes manually defined around the fetal brain. MSER regions of relevant size that fit within these boxes are used as positive examples whereas negative examples are taken further away from the fetal head.

Finally, a RANdom SAmple Consensus procedure (RANSAC, Fischler and Bolles, 1987) is used to find a best fitting cube around the fetal head. The rationale for fitting a cube is that the $2 \mathrm{D}$ detection process performs best in the central slices of the brain and that, due to the misalignment between slices, the detected 2D ellipses might not define a proper 3D ellipsoid. The dimensions of the 3D bounding cube are inferred from the gestational age and purposefully over-estimate the size of the brain by taking the $95^{\text {th }}$ centile of $O F D$ to ensure that the full brain will be processed during the segmentation step (Section 3.2). As the detection process does not provide information on the

orientation of the brain, the selected box is simply aligned with the image axes and is used to estimate the center of the brain in the 3D volume. The RANSAC procedure proceeds as follows: a restricted number of ellipses, typically 5, are randomly selected and their centroid used to place a box. A score is then computed by assessing the number of MSER regions fitting within that box. This process is repeated, typically 1000 times, and the box with the highest score is selected.

The result of the detection process is not only a bounding box, but also a partial segmentation of the brain corresponding to the selected MSER regions. Figure 2 shows an example of the resulting mask. It can be noted that the selected MSER regions do not cover the whole brain. In particular, due to the size selection using BPD and OFD, the extremal slices of the brain are unlikely to be included as they will be considered too small. The brain extraction method proposed in Section 3.2 is hence a propagation of this initial labeling within the region of interest defined by the detected bounding box.

\subsection{Brain extraction}

By using a 2D slice-by-slice patch-based segmentation we are able to propagate the initial labeling within the region of interest obtained from the detection process described in Section 3.1. A typical patch-based segmentation searches each region of the image to find the most similar patches within an atlas, a training database with known ground truth segmentation. The atlas is hence aligned with the image and a final segmentation is obtained by fusing the votes from selected patches of atlas images (Coupè et al., 2010; Rousseau et al., 2011).

In our application, we seek to segment an image using a sparse labeling of the same image. This sparse labeling corresponds to the periphery of the bounding box around the brain (background label), as well as the selected MSER regions (brain label), as shown in Figure 2. Therefore, we perform online learning in contrast to conventional atlas based approaches, which rely on offline training. Working on a single image at a time excludes the need to normalize intensities or register the volume to an atlas. Moreover, by learning from the same image, we can adapt to the variability of the unprocessed fetal MR data. In particular, we can work on $2 \mathrm{D}$ slices without prior knowledge of the scan orientation relative to the fetal anatomy. Similarly to Wang et al. (2013), we overcome the restriction of search windows by using a single global classifier that compares patches independently of their location in the image, instead of applying local classifiers. However, we chose a Random Forest classifier (Breiman, 2001), using 2D intensity patches as features, to maintain a feasible training and testing speed. A k-nearest neighbor classifier would have little training time, but its evaluation is a time-consuming process. The motivation for using $2 \mathrm{D}$ patches is that they are not corrupted by motion. Although the 2D slices are not necessarily parallel in the anatomy of the fetal brain, it can be expected that 2D patches small enough will share sufficient similarities across different regions of the brain to enable label propagation.

To obtain the initial sparse labeling on which the Random Forest is trained, the mask obtained from Section 3.1 is first 
preprocessed by drawing the ellipses fitted to the MSER regions and filling any remaining hole. These regions provide positive examples of $2 \mathrm{D}$ patches to learn the appearance of the fetal brain, while negative examples are obtained by extracting patches on a narrow band at the boundary of the detected bounding box (Figure 2.b). The resulting classifier is then applied to the volume within the detected bounding box, to obtain a probability map of brain tissues.

An estimation of the volume of the fetal brain from the gestational age (Chang et al., 2003) is used to refine the probabilistic output of the Random Forest. This can be interpreted as a volume constraint in the segmentation process. For this purpose, enough of the voxels most likely to be within the brain are selected in the probability map to fill half of the estimated volume of the brain, and subsequently set to the highest probability value. Likewise, enough of the voxels most likely to be part of the background are selected to fill half of the background, and set to the smallest probability value. The probability map is then rescaled between $[0,1]$. This corresponds to trimming both sides of the intensity histogram of the probability map before rescaling it.

The probability map is then converted to a binary segmentation using a Conditional Random Field (CRF, Lafferty et al., 2001). This is a common approach in computer vision which enables the combination of local classification priors (unary term) with a global consistency constraint (pairwise term). We use the formulation proposed by Boykov and Funka-Lea (2006) which seeks to minimize the energy function:

$$
E(l)=\sum_{p} D\left(p, l_{p}\right)+\lambda \sum_{p, q} V_{p q}\left(l_{p}, l_{q}\right)
$$

with the unary term (data cost) defined by the probabilistic prediction of the Random Forest classifier:

$$
D\left(p, l_{p}\right)=-\log \left(\mathrm{P}\left(p \mid l_{p}\right)\right)
$$

and a pairwise term (smoothness cost) penalizing labeling discontinuities between pixels of similar intensities:

$$
V_{p q}\left(l_{p}, l_{q}\right)=\frac{\left[l_{p} \neq l_{q}\right]}{\|p-q\|_{2}} e^{-\left(I_{p}-I_{q}\right)^{2} / 2 \sigma^{2}}
$$

where $\|p-q\|_{2}$ is the distance between pixels taking image sampling into account, $\left|I_{p}-I_{q}\right|$ the intensity difference between pixels and $\sigma$ an estimation of the image noise. $\lambda$ is a weighting between the data cost and the smoothness cost. High values of $\lambda$ will tend to assign the same label to large connected components while small values of $\lambda$ will tend to preserve the initial labeling. This definition of the pairwise term is the same as the edge weight of the graph cut used by Anquez et al. (2009) for the segmentation of the fetal brain. An estimation of the image noise is obtained by computing the difference between the image and a Gaussian blurred version of the image. A more robust method that would keep image edges from contributing to the noise estimate could be considered in future work. When building the CRF, each voxel is connected to its 26 neighbors with an edge of cost $V_{p q}$. In order to take fetal motion into account, we compute distinct values of $\sigma$ for in-plane and through-plane
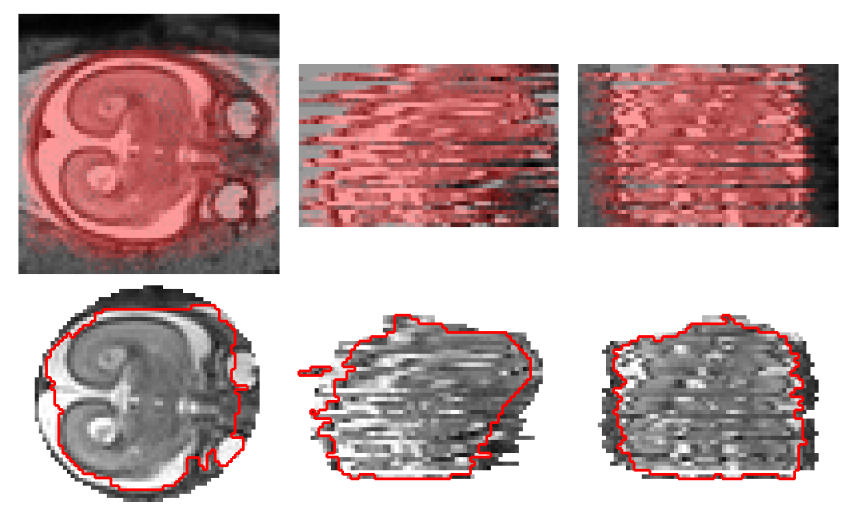

Figure 3: The top row shows a probability map of the Random Forest after rescaling while the bottom row shows the segmentation from the CRF (red contour) enlarged with 2D ellipses.

edges of the CRF, namely $\sigma_{x y}$ is computed using an in-plane Gaussian blurring while $\sigma_{z}$ is computed using a through-plane Gaussian blurring.

Whereas the Random Forest classifier previously described was applied on 2D slices, we rely on a 3D CRF to force the extraction of a blob-like structure, thus minimizing the error in the extremal slices of the brain. To ensure that enough structures of the fetal skull remain in the segmentation as a guide in the registration process, ellipses are fitted on every 2D slice of the segmentation mask and made $10 \%$ larger on the final segmentation mask.

The resulting segmentation, as shown in Figure 3, is then used as input for the motion correction procedure. In cases of extreme motion, it could be considered to treat each slice independently with a $2 \mathrm{D} \mathrm{CRF}$, thus allowing for more discontinuities in the segmented structure. If there is little motion between stacks, a 4D CRF could be considered, the fourth dimension being the time between stack acquisitions, thus taking advantage of the overlap between images.

\subsection{Joint segmentation and motion correction}

The automated masking of the fetal brain has been integrated with the motion correction method of Kuklisova-Murgasova et al. (2012). The latter performs slice-to-volume registration interleaved with a super-resolution scheme and robust statistics to remove poorly registered or corrupted slices. More specifically, the original slices are classified into inliers and outliers based on their similarity with the reconstructed volume. This process seeks to remove artifacts of motion corruption and misregistration. We propose to integrate automatically generated masks for all input stacks instead of a manual input. Moreover, we are able to refine the segmentation in the final iterations of the motion correction procedure.

The motion correction process is initialized as shown in Figure 4. Before performing any cropping, all stacks are registered to a template stack in order to correct for maternal motion. This template stack is typically chosen manually as the one least affected by fetal motion. The same template stack was used for 


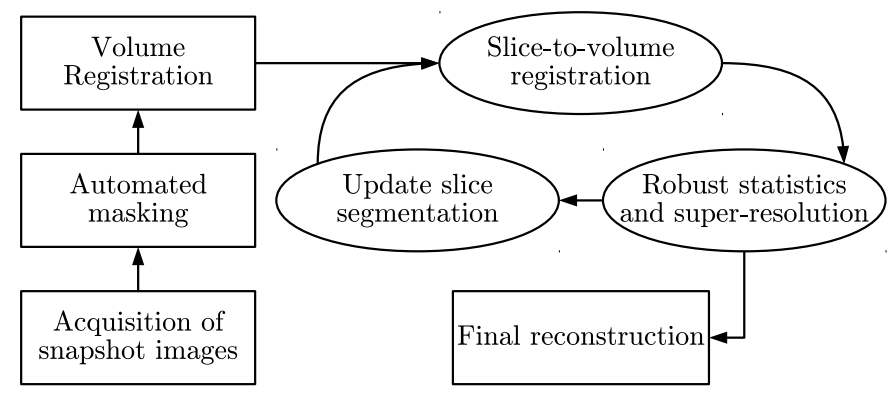

Figure 4: The masks of the fetal brain for the original 2D slices are progressively refined using the updated slice-to-volume registration transformations.

the manual and automated initializations during testing (Section 5.2). All stacks are then automatically masked and subsequently registered to the template stack for a second time. A large mask encompassing all masks is then created to ensure that the brain is not cropped. Indeed, each segmentation mask will have more confidence in the central slices of the brain than in the extremal slices. This mask is then updated after each iteration of the slice-to-volume registration. This approach significantly differs from Kuklisova-Murgasova et al. (2012) who use a static mask from the template stack, but it can be related to the evolving mask used by Kim et al. (2010).

As the reconstruction progresses, the segmentation of the original slices can be refined thanks to their recovered alignment in 3D space. In the final iterations of motion correction, the boxes obtained in Section 3.1 are used without applying any mask in order to obtain a reconstructed volume larger than the brain. The slice-by-slice probability maps obtained in Section 3.2 that have been classified as inliers with a strong confidence are then selected and summed to obtain a 3D probability map (Figure 5). Following the same method described in Section 3.2, the latter is then rescaled using an expected brain volume and used to initialize a 3D CRF on the enlarged reconstructed volume. Finally, the resulting mask is smoothed to avoid boundary artifacts, and dilated to incorporate skull tissues.

This mask can then be projected back to the original slices and thus correct initial errors. As the only information that is passed on from one iteration to the next are rigid transformations, the motion correction can proceed with the updated masked slices (Figure 4). This 3D CRF is only applied in the final iterations as the motion correction starts from a low resolution estimate of the reconstructed volume and progressively produces a sharper result.

\section{Implementation}

To gain prior knowledge about the expected size of the brain, we used a 2D ultrasound study from Snijders and Nicolaides (2003) performed on 1040 normal singleton pregnancies. The $5^{\text {th }}$ and $95^{\text {th }}$ centile values of the occipitofrontal diameter and the biparietal diameter have been used to define the acceptable size and aspect ratio for the selected MSER regions (Sec-
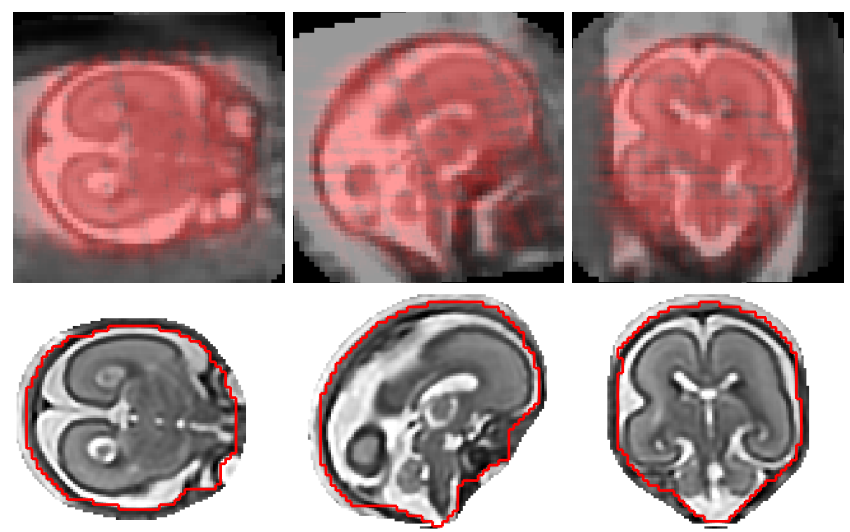

Figure 5: The whole boxes detected in Section 3.1 are used without any masking to reconstruct a volume larger than the brain. The probability maps obtained in Section 3.2 are then combined to initialize a CRF (top row). The bottom row presents the final reconstruction with the CRF segmentation in red.

tion 3.1). In addition, the brain volume in Section 3.2 is estimated from a 3D ultrasound study carried out by Chang et al. (2003) on 203 singleton fetuses with gestational ages between 20 and 40 weeks.

The parameters of the proposed methods are $N$, the size of the vocabulary in the Bag-of-Words model of the detection process, $\lambda$, the weight between spatial constraint and prior knowledge in the CRF segmentation, the patch size and the number of trees in the label propagation. Following the experiments of Fulkerson et al. (2009), the size $N$ of the Bag-of-Words vocabulary has been set to 400 . The remaining parameters have been chosen empirically, that is to say seeking to optimize the results on a few images by trial and error. In the 3D CRF, the weight $\lambda$ has been set to 20 in Section 3.2 and 10 in Section 3.3. The performance of the segmentation is fairly stable across a wide range of values for the number of trees and the patch size, and the choice then becomes motivated by preserving the speed of the method, which decreases with higher values of these parameters. We chose to use a patch size of $17 \times 17$ pixels (see Figure 2.b) and Random Forests of 30 trees in our patch-based segmentation. While the motion correction reconstructs a volume with a resolution of $0.75 \mathrm{~mm}$, the CRF run in the last steps of motion correction is performed after downsampling the reconstructed volume with a factor 2 to limit memory consumption, hence at $1.5 \mathrm{~mm}$ resolution.

The code was implemented with the IRTK library, using OpenCV (Bradski, 2000) and scikit-learn (Pedregosa et al., 2011) for the detection and segmentation parts, and is available online $^{1}$. The average time measurement for the separate steps of the pipeline is shown in Table 1. While the brain detection and extraction can be run on a normal PC, motion correction was performed on a 24 core, 64 GB RAM computer. A GPU accelerated version of the motion correction method, which runs within 10min per subject, is under development.

\footnotetext{
${ }^{1}$ https://github.com/BioMedIA/IRTK
} 


\begin{tabular}{lr}
\hline Step & Time \\
\hline brain localization & $<1 \mathrm{~min}$ \\
brain extraction & $2 \mathrm{~min}$ \\
motion correction & $1 \mathrm{~h} 20$ \\
3D CRF & $10 \mathrm{~min}$ \\
\hline
\end{tabular}

Table 1: Average computation time for the separate steps of the pipeline.

\section{Evaluation}

\subsection{Data acquisition}

The data used for evaluating the proposed method is the same as in Keraudren et al. (2013). Ethical approval was granted by the Hammersmith Hospital Ethics Committee (ethics no. 97/H0707/105). Fetal MRI was performed on a 1.5T Philips Achieva MRI system using a 32 channel cardiac array for signal reception. Stacks of T2-weighted dynamic ssTSE images were obtained with the following scanning parameters: TR $15000 \mathrm{~ms}$, TE $160 \mathrm{~ms}$, slice thickness of $2.5 \mathrm{~mm}$, slice overlap of $1.25 \mathrm{~mm}$, flip angle $90^{\circ}$. The in-plane resolution is $1.25 \mathrm{~mm}$. There were 50-100 slices per stack divided into 4-6 interleaved packets, with one packet acquired within a single TR. Acquisition times are about 1-2min per stack, all stacks being acquired within 15-20min. A parallel imaging speed up SENSE factor of 2 was applied in all stacks. Data from 59 healthy fetuses with gestational age ranging from 22 to 39 weeks were used in this study, with 5 fetuses scanned twice and one three times, amounting to a total of 66 datasets. Each dataset consists of 3 to 8 stacks of slices acquired in three standard orthogonal planes, representing a total of 117 sagittal, 113 coronal and 228 transverse scans.

During the localization process, standardizing the image contrast is crucial as both MSER and SIFT features depend on gradient information. The data has thus been contrast-normalized over all slices, with saturation of $1 \%$ on each side of the intensity histogram. The acquired slices have been upsampled to $0.8 \times 0.8 \mathrm{~mm}$ resolution to increase the number of SIFT features detected as well as to limit the variation in scale between datasets.

\subsection{Ground truth and experiments}

The automated brain extraction was evaluated using a tenfold cross validation: 39 subjects were randomly selected for training the brain detector described in Section 3.1 and 20 subjects for testing the automated slice-by-slice segmentation, and this process was repeated 10 times. Fetuses who have been scanned several times have not been separated during cross validation to avoid having similar datasets for both training and testing. The Bag-of-Words classifier of the brain detector was trained using bounding boxes tightly drawn around the brain.

A motion corrected 3D image reconstruction was generated for each subject using an automated brain masking randomly selected from one instance of the ten-fold experiment.

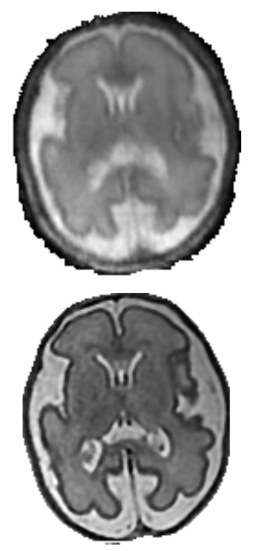

(a)

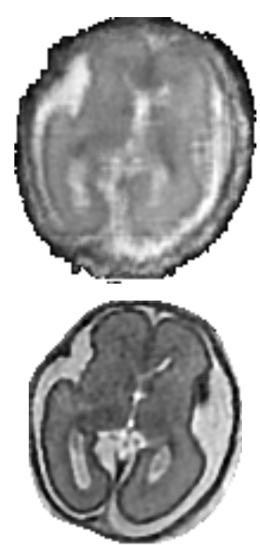

(b)
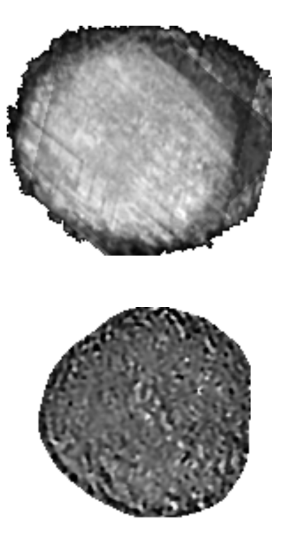

(c)
Figure 6: The success of the motion correction procedure is strongly dependent on the initial volume-to-volume registration. For each pair of images, the top row presents the initial average of all stacks while the bottom row is the final reconstruction. Column (a) is an example with almost no motion, column (b) is an example with motion that is successfully corrected and column (c), where the brain is barely recognizable in the average image, is an example with extreme motion where motion correction fails.

These were evaluated against reconstructions performed with the same algorithm, but with a manual mask drawn on one stack and propagated to the other stacks after rigid alignment. The 3D motion corrected reconstructions achieved using manual brain extraction were treated as a gold standard and used to directly evaluate the proposed segmentation pipeline for the fetal brain. This was done in two ways:

Firstly an expert observer, blinded to the reconstruction method, viewed both reconstructions for each subject in random order and rated them using the following qualitative scale:

A. this reconstruction has no evident artifacts from motion.

B. this reconstruction has minor artifacts but it can still be used for diagnosis.

C. this reconstruction cannot be used for clinical diagnosis (failed motion correction).

The same expert was also asked to assign a motion score to each subject based on the appearance of the initial average of all stacks after volume-to-volume registration and before any sliceto-volume registration. In case of doubt between severe motion and low SNR, the expert looked at the original scan acquisitions in order to assign a score. The scores were assigned as follows:

I. no motion (Figure 6.a).

II. some motion (Figure 6.b).

III. severe motion (Figure 6.c).

Secondly, the volumetric images generated starting with manual brain extraction were segmented using the patch-based method of Wright et al. (2012). The rigid body transformations determined in aligning the original slices with the reconstructed volume were then used to project the ground truth segmentation 


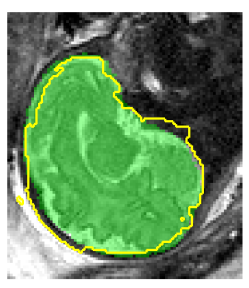

(a)

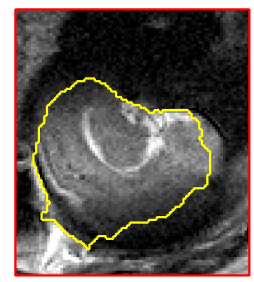

(b) (c)

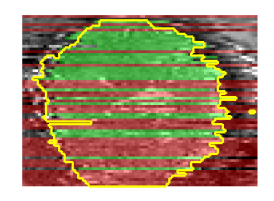

Figure 7: Example of ground truth segmentation (green), slices excluded from motion correction (red) and automated segmentation (yellow). (a) and (b) are original 2D slices while (c) is a through-plane cut. (a) has been classified as inlier during motion correction and (b) as outlier due to motion artifacts.

onto the original slices. The motion correction method removes artifacts of motion corruption and misregistration by classifying the original slices into inliers and outliers (KuklisovaMurgasova et al., 2012) based on their similarity with the reconstructed volume. Only slices which have been included in the motion corrected volume with a strong confidence were used to evaluate the slice-by-slice segmentation (Figure 7). Also, subjects whose motion correction using manual masks failed have been excluded from the slice-by-slice evaluation, as no ground truth could be generated.

When localizing and segmenting the fetal brain, normal growth charts are used to define a realistic range of sizes and volumes of the brain in order to remove improbable detection results, while still allowing a large variation in brain size. From standard growth charts, extending to include $99.6 \%$ of subjects corresponds to a 1 week error. To assess the stability of the proposed methods depending on the age input, we thus performed a separate ten-fold cross validation simulating extreme growth restriction by adding a 5 week error in all gestational ages.

Additionally, the results obtained using the method of Taleb et al. (2013), which is part of the Baby Brain Toolkit (Rousseau et al., 2013), are also reported.

\subsection{Evaluation metrics}

The proposed segmentation pipeline for the fetal brain was evaluated using the measures of recall and precision (Baeza-Yates and Ribeiro-Neto, 1999). The recall, defined as true positives, measures in our case how much of the true positives + false negatives,
brain is correctly segmented:

$$
\text { recall }=\frac{\text { Volume of correctly classified voxels }}{\text { Ground truth volume of the brain }}
$$

while the precision, defined as $\frac{\text { true positives }}{\text { true positives + false positives }}$, gives an estimate of how much of the background is correctly excluded:

$$
\text { precision }=\frac{\text { Volume of correctly classified voxels }}{\text { Detected volume of the brain }}
$$

Similarly to Ison et al. (2012), we selected these two metrics as they provide a mean to compare the extraction of the brain in its entirety while minimizing the inclusion of other tissues.

\begin{tabular}{|c|c|c|}
\cline { 2 - 3 } \multicolumn{1}{c|}{} & $\begin{array}{c}\text { Mean } \\
\text { recall }\end{array}$ & $\begin{array}{c}\text { Mean } \\
\text { precision }\end{array}$ \\
\hline Box detection & $98.8( \pm 2.8)$ & $57.0( \pm 7.7)$ \\
\hline RF/CRF segmentation & $90.6( \pm 9.7)$ & $90.3( \pm 9.5)$ \\
\hline Enlarged segmentation & $96.4( \pm 9.4)$ & $73.3( \pm 9.0)$ \\
\hline Final segmentation & $93.2( \pm 3.7)$ & $93.0( \pm 5.1)$ \\
\hline \hline Taleb et al. & $93.1( \pm 17.4)$ & $68.7( \pm 19.8)$ \\
\hline
\end{tabular}

Table 2: Mean recall and mean precision ( \pm standard deviation) computed over the ten-fold cross validation and for the reconstructed volumes.

Moreover, these measures have the advantage of being independent of the amount of background and are thus not biased when images are cropped. The segmentation we seek as input for motion correction should have a maximal recall as it should contain the whole brain. However, its precision should be high enough for registration to succeed thanks to the exclusion of maternal tissues, but not necessarily maximal as including tissues of the fetal head can help the registration. Additionally, we present Dice segmentation scores (Dice, 1945) which summarize both recall and precision measures:

$$
\mathbf{d}=2 \times \frac{|A \cap B|}{|A|+|B|}=2 \times \frac{\text { precision } \times \text { recall }}{\text { precision }+ \text { recall }}
$$

where $|A|$ and $|B|$ represent respectively the detected and ground truth volume of the brain.

\subsection{Localization and segmentation results}

The density plots in Figure 8 summarize the distribution of the recall and precision scores at different stages of the workflow. The box detection corresponds to the localization of the fetal brain. The $R F / C R F$ segmentation is the extracted brain after the patch-based Random Forest and CRF segmentation, while the enlarged segmentation is the same segmentation after dilation. Lastly, the final segmentation is the segmentation of the motion corrected brain. Table 2 presents the mean values for these scores.

The automated detection of a bounding box for the fetal brain (Section 3.1) contains in average more than $98.8 \%$ of the brain. The mean distance between the center of the detected box and the ground truth bounding box is $5.1 \mathrm{~mm}( \pm 2.6 \mathrm{~mm})$. However, the detected box encompasses a large portion of background pixels, with a precision of only $57.0 \%$. This low score can be expected as we select a box whereas the shape of the brain is ellipsoidal, and we purposefully overestimate the dimensions of that box (Section 3.1).

We therefore segment the content of the detected box into brain and non-brain tissues. The CRF segmentation has both recall and precision mean scores above $90 \%$. However, this segmentation misses parts of the brain and does not include tissues of the fetal skull, which are potentially useful for registration. We hence enlarge it, increasing the recall score as more brain tissues are included $(96.4 \%)$, but decreasing the precision score as more background voxels are also included (73.3\%). 


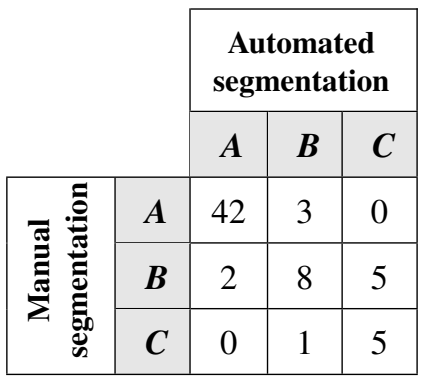

(a)

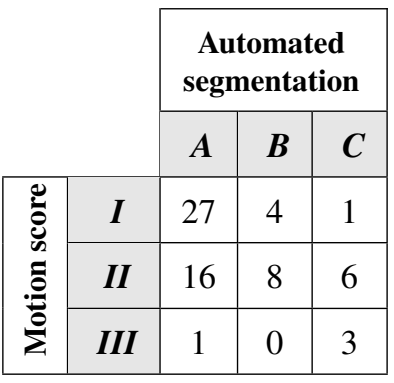

(b)
Table 3: Table (a) presents the quality scores obtained for each subject for the automated and manual initialization of motion correction. The diagonal corresponds to subjects where both methods gave results of similar quality, while the upper part of the array corresponds to automated initializations with a higher quality score and the lower part corresponds to manual initializations with a higher score. Table (b) presents the quality scores of the automated initialization depending on the motion scores. It can be noted that most cases with little motion were successfully reconstructed while most cases with extreme motion failed.

This enlarged segmentation is used as input for motion correction, as well as the $2 \mathrm{D}$ probability maps obtained at the $R F / C R F$ segmentation stage. The detailed segmentation we obtain after motion correction has both mean recall and mean precision scores above $93 \%$. This segmentation improves the robust statistics performed during motion correction, leading to a sharper motion corrected volume (Figure 9).

Simulating extreme growth restriction by adding a 5 week error in all gestational ages, the mean Dice score of the $R F / C R F$ segmentation (Section 3.2) in the ten-fold cross-validation is still $86.7 \%$, which should be compared with the mean Dice score of $90.5 \%$ obtained when providing correct estimates of gestational ages.

The masks produced using the method of Taleb et al. (2013) obtained a mean Dice score of $80.4 \%( \pm 16.2 \%)$. These masks should be compared with the enlarged segmentation masks produced by the proposed method and that are used as input for motion correction, which obtained a mean Dice score of $84.2 \%$ $( \pm 8.2 \%)$. The longer tail of the precision distribution in the rightmost plot of Figure 8 highlights the higher number of unsuccessful localizations of the brain using the method of Taleb et al. (2013).

\subsection{Reconstruction results}

The results of the evaluation of motion correction are presented in Table 3.a. It can be noted that in $88 \%$ of cases, the automated initialization scored similarly or better than the manual initialization, and in $85 \%$ of cases the reconstructed volume obtained from the automated initialization was good enough for clinical diagnosis. Although the automated initialization failed to produce meaningful results in $15 \%$ of cases, neither methods produced useful results in $7.5 \%$ of cases. This is mostly due to
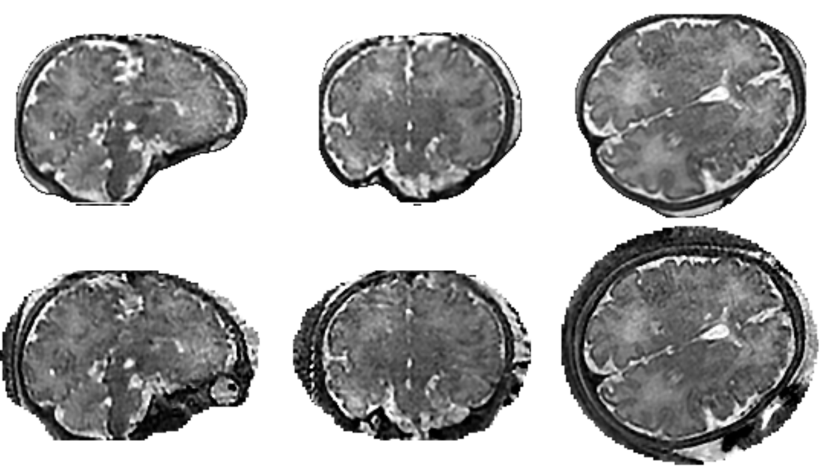

Figure 9: Example reconstruction with automated initialization (top row) and manual initialization (bottom row). It can be observed that the method with automated segmentation produces a tighter mask of the brain, thus resulting in less artifacts.

a poor initial alignment of the stacks (Figure 6.c), discussed in Section 6.

Among the 6 subjects that received a quality score of $\mathrm{C}$ for the manual initialization (Table 3.a), we excluded from the ground truth dataset the 4 cases corresponding to registration failures while keeping the 2 cases corresponding to low SNR. In a few cases $(4.5 \%)$, the automated method performed better than the manual segmentation. An example is presented in Figure 9 and can be explained by the tighter mask produced by the automated method.

Table 3.b presents the motion scores assigned by the expert. These scores give an overview of the amount of motion in our data, with $48 \%$ of subjects classified as no motion, $45 \%$ as some motion and $6 \%$ as severe motion. The case with a quality score of $\mathrm{C}$ and a motion score of $\mathrm{I}$ has a very low signal-to-noise ratio (SNR) and the default parameters of motion correction would need to be adjusted to perform an adequate reconstruction. It can be noted that cases with extreme motion represent only a small portion of our dataset but that our method did not enable a successful motion correction of most of these cases.

\section{Discussion}

The scores for the $R F / C R F$ segmentation and the enlarged segmentation can be compared with the sensitivity (recall) and specificity (precision) scores reported by Ison et al. (2012), which were respectively between $74 \%$ and $88 \%$, and between $85 \%$ and $89 \%$, depending on the scan orientation (sagittal, coronal or transverse). The mean Dice score for the final segmentation is $93.0 \%$, thus providing a good starting point for processing the fetal brain after motion correction. Brain masks before motion correction were obtained with the method of Taleb et al. (2013) for the datasets used in this paper and compared to the proposed pipeline through mean Dice scores. Our proposed method, which relies on a slice-by-slice detection and segmentation, is thus more robust than the registration based approach of Taleb et al. (2013).

As can be seen from Figure 8, the box detection succeeded for all stacks, but a few stacks (less than 1\%) failed to be seg- 


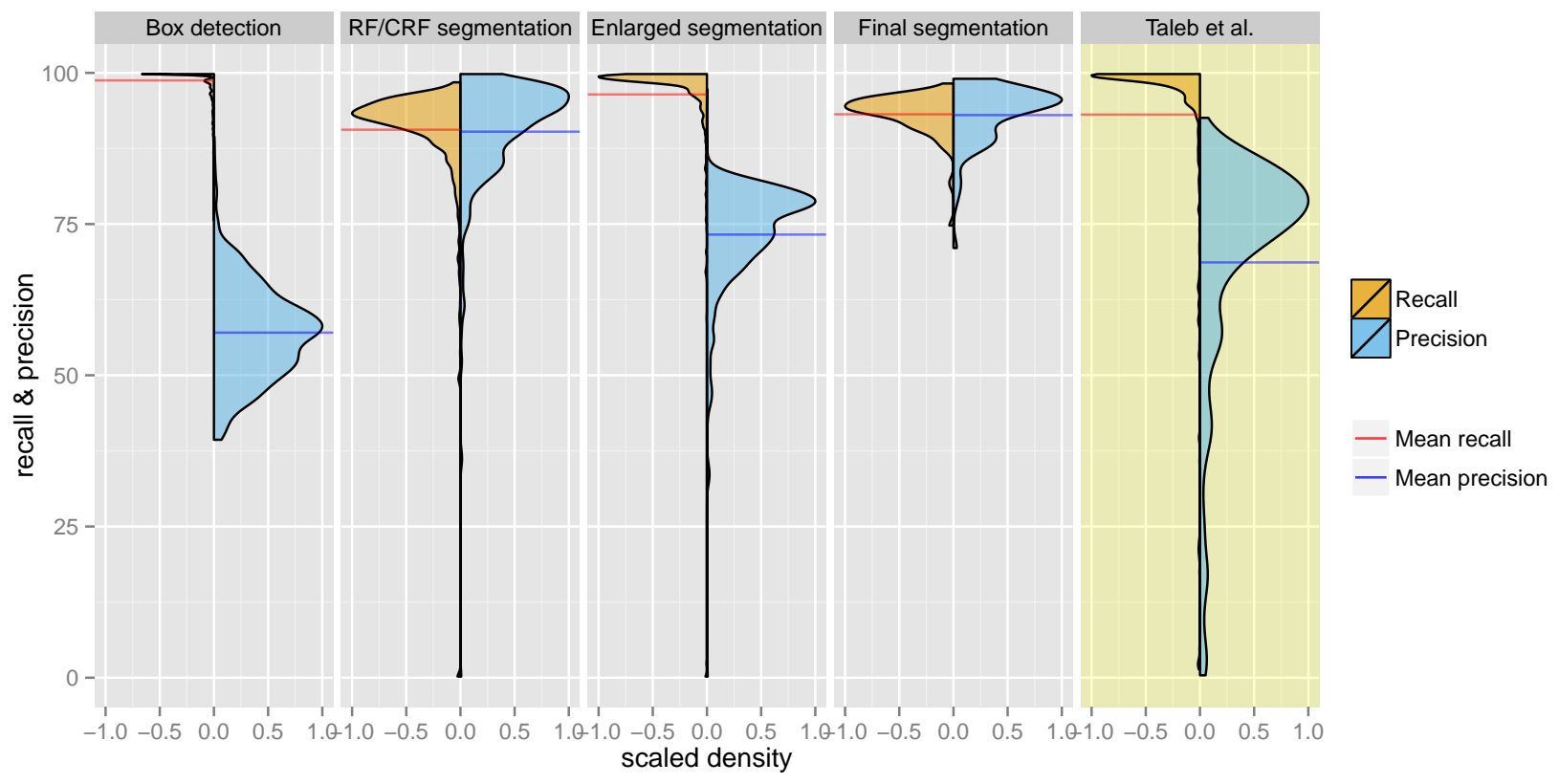

Figure 8: The localization of the brain in Section 3.1 has a high recall but a low precision (Box detection): it covers the whole brain but does not discriminate the background well. The subsequent Random Forest and CRF segmentation provides a better precision but has a lower recall as it misses fragments of the brain $(R F / C R F$ segmentation). This segmentation is hence enlarged by fitting $2 \mathrm{D}$ ellipses in order to include as much brain and skull tissues as possible, thus increasing the recall and decreasing the precision (Enlarged segmentation). The Final segmentation is the precise segmentation of the brain obtained when completing motion correction. The rightmost plot presents the brain extraction results obtained using the method of Taleb et al. (2013) and should be compared with the results from the Enlarged segmentation ( $\left.3^{\text {rd }} \mathrm{plot}\right)$.

mented. This does not necessarily mean that manual intervention will be required in order to perform motion correction. Indeed, assuming that most of the stacks from the same subject are correctly segmented, the motion correction procedure then automatically rejects wrongly segmented stacks based on their dissimilarity with the rest of the dataset.

This demonstrates the success of the proposed method: it allows a full automatization of motion correction, saving the time spent on manual segmentation while still providing comparable results. Moreover, it can be noted that the masks resulting from the automated initialization are of similar quality in terms of brain coverage and exclusion of external tissues while the manual masks have more variability, sometimes not fully covering all intracranial structures or including too much background, mostly due to methodological differences between experts rather than to the fetus position or motion.

Among the 5 subjects that failed to be reconstructed with the automated method but were successfully reconstructed with the manual initialization, 3 failures can be attributed to part of the maternal bladder or portions of amniotic fluid between the fetal head and the uterine wall being included in the segmentation. This is a weakness inherent to our detection method. As these regions are of homogeneous intensity, they are selected in the MSER detection process. Furthermore, as the ellipses fitted to them encompass the whole brain, they are kept in the Bag-ofWords classification. The resulting segmentation is a mask too wide for motion correction to be efficient. For the remaining 2 cases, the segmentation missed part of the brain, which could have been improved by setting a lower $\lambda$ value in the CRF segmentations, namely a weaker smoothness constraint.

Abnormal brain growth resulting in a smaller brain was simulated by altering the gestational ages in our dataset. As can be expected from the filtering by size taking place during the brain detection process, the method showed some sensitivity to abnormal sizes of the brain, with the mean Dice score of the $R F / C R F$ segmentation decreasing by $3.8 \%$. However, it can be expected that brains with a very abnormal structure, in conditions such as severe ventriculomegaly or hydrocephalus, will not be segmented with the proposed method without including subjects with similar pathologies in the training dataset. Indeed, for the trained classifier in the detection process to be able to generalize from the training data to any unseen subject, this training data needs to be representative of the possible test cases.

As presented in Figure 6, the success of motion correction strongly depends on the initial alignment of all stacks after volume-to-volume registration. If the initial misalignment is too significant, the slice-to-volume registration, which performs a spatially limited search, will not be able to correct it. This highlights the main limitation of the proposed method: the orientation of the brain is still unknown after segmentation and the initial alignment between stacks is left to an intensity based image registration, which is initialized using the scanner world coordinate system. 
The whole process could be further improved if information obtained from the segmentation was used more extensively in the alignment process. Likewise, the robust statistics that remove poorly registered or corrupted slices (Section 3.3) are only based on intensities and could gain in accuracy if combined with the segmentation.

\section{Conclusion}

In this paper we have presented a method to automatically localize, segment, and refine the segmentation of the fetal brain from misaligned 2D slices, making manual effort obsolete for fetal brain MRI reconstruction.

The proposed method has been evaluated on a large database of 66 subjects and when assessed by an expert, it obtained in $88 \%$ of cases a quality score similar or better to motion correction performed using manual segmentation.

In future work, our method would only necessitate minor modifications to be applied to fetal functional MRI. In particular, the Bag-of-Words with SIFT features for localizing the brain would need to be trained on functional MRI data due to the difference in intensity patterns. The proposed method has the advantage of only learning from a bounding box ground truth, which is simple to obtain compared to a detailed segmentation. Nonetheless, knowledge of the appearance of the rest of the fetal head could be beneficial in order to obtain a more precise segmentation that would preserve more facial tissues while excluding maternal tissues, enabling a more robust initial registration.

A fully automated pipeline for motion correction of the fetal brain in MRI paves the way for both clinical use and larger scale research studies. It is a key step to widespread uptake of 3D fetal imaging from robust, motion tolerant snapshot methods.

\section{Acknowledgments}

Kevin Keraudren was supported by the EPSRC grant EP/H046410/1. Maria Murgasova was supported by the MRC strategic grant MR/K006355/1. Bernhard Kainz was supported by a Marie Curie Intra-European Fellowship within the $7^{\text {th }}$ European Community Framework Programme (FP7-PEOPLE2012-IEF F.A.U.S.T. 325661).

The research was funded/supported by the National Institute for Health Research (NIHR) Biomedical Research Centre based at Guy's and St Thomas' NHS Foundation Trust and King's College London (reference guysbrc-2012-1). The views expressed are those of the authors and not necessarily those of the NHS, the NIHR or the Department of Health.

\section{References}

Anquez, J., Angelini, E.D., Bloch, I., 2009. Automatic segmentation of head structures on fetal mri, in: Proceedings of the Sixth IEEE International Conference on Symposium on Biomedical Imaging: From Nano to Macro, IEEE Press, Piscataway, NJ, USA. pp. 109-112.

Baeza-Yates, R.A., Ribeiro-Neto, B., 1999. Modern Information Retrieval. Addison-Wesley Longman Publishing Co., Inc., Boston, MA, USA.
Boykov, Y., Funka-Lea, G., 2006. Graph Cuts and Efficient N-D Image Segmentation. Int. J. Comput. Vision 70, 109-131. doi:10.1007/ s11263-006-7934-5.

Bradski, G., 2000. The OpenCV Library. Dr. Dobb's Journal of Software Tools 25, 120-126.

Breiman, L., 2001. Random Forests. Mach. Learn. 45, 5-32. doi:10.1023/A : 1010933404324.

Chang, C.H., Yu, C.H., Chang, F.M., Ko, H.C., Chen, H.Y., 2003. The Assessment of Normal Fetal Brain Volume by 3-D Ultrasound. Ultrasound in Medicine \& Biology 29, 1267-1272. doi:10.1016/S0301-5629(03) 00989-X.

Chen, Q., Levine, D., 2001. Fast Fetal Magnetic Resonance Imaging Techniques. Topics in Magnetic Resonance Imaging 12, 67.

Coupè, P., Manòn, J.V., Fonov, V., Pruessner, J., Robles, M., Collins, D.L., 2010. Nonlocal Patch-Based Label Fusion for Hippocampus Segmentation, in: Jiang, T., Navab, N., Pluim, J., Viergever, M. (Eds.), MICCAI'10. Springer Berlin Heidelberg. volume 6363 of LNCS, pp. 129-136. doi:10.1007/978-3-642-15711-0_17.

Csurka, G., Dance, C., Fan, L., Willamowski, J., Bray, C., 2004. Visual Categorization With Bags of Keypoints, in: Workshop on Statistical Learning in Computer Vision, ECCV, pp. 6-22.

Dice, L.R., 1945. Measures of the Amount of Ecologic Association Between Species. Ecology 26, 297-302. doi:10.2307/1932409.

Donoser, M., Bischof, H., 2006. 3D Segmentation by Maximally Stable Volumes (MSVs), in: International Conference on Pattern Recognition ICPR'06, IEEE Computer Society. pp. 63-66.

Eskildsen, S.F., Coupé, P., Fonov, V., Manjón, J.V., Leung, K.K., Guizard, N., Wassef, S.N., Østergaard, L.R., Collins, D.L., 2012. BEaST: Brain Extraction based on Nonlocal Segmentation Technique. NeuroImage 59, 2362 2373. doi:10.1016/j.neuroimage. 2011.09.012.

Fischler, M.A., Bolles, R.C., 1987. Random Sample Consensus: A Paradigm for Model Fitting with Applications to Image Analysis and Automated Cartography, in: Fischler, M.A., Firschein, O. (Eds.), Readings in Computer Vision: Issues, Problems, Principles, and Paradigms. Morgan Kaufmann Publishers Inc., San Francisco, CA, USA, pp. 726-740.

Fulkerson, B., Vedaldi, A., Soatto, S., 2009. Class Segmentation and Object Localization with Superpixel Neighborhoods, in: ICCV'09, IEEE. pp. 670677.

Gholipour, A., Estroff, J., Warfield, S., 2010. Robust Super-Resolution Volume Reconstruction From Slice Acquisitions: Application to Fetal Brain MRI. Medical Imaging, IEEE Transactions on 29, 1739-1758. doi:10. 1109/TMI . 2010.2051680.

Ison, M., Donner, R., Dittrich, E., Kasprian, G., Prayer, D., Langs, G., 2012. Fully Automated Brain Extraction and Orientation in Raw Fetal MRI, in: Proc. Workshop on Paediatric and Perinatal Imaging, MICCAI'12, pp. 1724.

Jiang, S., Xue, H., Glover, A., Rutherford, M., Rueckert, D., Hajnal, J., 2007. MRI of Moving Subjects Using Multislice Snapshot Images With Volume Reconstruction (SVR): Application to Fetal, Neonatal, and Adult Brain Studies. Medical Imaging, IEEE Transactions on 26, 967-980. doi:10.1109/TMI . 2007.895456.

Kainz, B., Keraudren, K., Kyriakopoulou, V., Rutherford, M., Hajnal, J., Rueckert, D., 2014. Fast Fully Automatic Brain Detection in Fetal MRI Using Dense Rotation Invariant Image Descriptors, in: ISBI'14, IEEE. pp. 1230-1233.

Keraudren, K., Kyriakopoulou, V., Rutherford, M.A., Hajnal, J.V., Rueckert, D., 2013. Localisation of the Brain in Fetal MRI Using Bundled SIFT Features., in: Mori, K., Sakuma, I., Sato, Y., Barillot, C., Navab, N. (Eds.), MICCAI'13, Part I, Springer Berlin Heidelberg. pp. 582-589.

Kim, K., Habas, P., Rousseau, F., Glenn, O., Barkovich, A., Studholme, C., 2010. Intersection Based Motion Correction of Multislice MRI for 3-D in Utero Fetal Brain Image Formation. Medical Imaging, IEEE Transactions on 29, 146-158. doi:10.1109/TMI .2009.2030679.

Kuklisova-Murgasova, M., Quaghebeur, G., Rutherford, M.A., Hajnal, J.V., Schnabel, J.A., 2012. Reconstruction of Fetal Brain $\{M R I\}$ with Intensity Matching and Complete Outlier Removal. Medical Image Analysis 16, 1550 - 1564. doi:10.1016/j.media.2012.07.004.

Lafferty, J.D., McCallum, A., Pereira, F.C.N., 2001. Conditional Random Fields: Probabilistic Models for Segmenting and Labeling Sequence Data, in: Proceedings of the Eighteenth International Conference on Machine Learning, Morgan Kaufmann Publishers Inc., San Francisco, CA, USA. pp. 
Malamateniou, C., Malik, S., Counsell, S., Allsop, J., McGuinness, A., Hayat, T., Broadhouse, K., Nunes, R., Ederies, A., Hajnal, J., et al., 2013. MotionCompensation Techniques in Neonatal and Fetal MR Imaging. American Journal of Neuroradiology 34, 1124-1136. doi:10.3174/ajnr . A3128.

Matas, J., Chum, O., Urban, M., Pajdla, T., 2004. Robust Wide-Baseline Stereo from Maximally Stable Extremal Regions. Image and Vision Computing 22, 761 - 767. doi:10.1016/j.imavis.2004.02.006. british Machine Vision Computing 2002.

Najman, L., Couprie, M., et al., 2004. Quasilinear Algorithm for the Component Tree, in: SPIE, pp. 98-107. doi:10.1117/12.526592.

Pedregosa, F., Varoquaux, G., Gramfort, A., Michel, V., Thirion, B., Grisel, O., Blondel, M., Prettenhofer, P., Weiss, R., Dubourg, V., Vanderplas, J., Passos, A., Cournapeau, D., Brucher, M., Perrot, M., Duchesnay, E., 2011. Scikitlearn: Machine Learning in Python. Journal of Machine Learning Research 12, 2825-2830.

Rousseau, F., Glenn, O., Iordanova, B., Rodriguez-Carranza, C., Vigneron, D., Barkovich, J., Studholme, C., et al., 2006. Registration-based Approach for Reconstruction of High Resolution In Utero Fetal MR Brain Images. Academic Radiology 13, 1072-1081. doi:10.1016/j . acra.2006.05.003.

Rousseau, F., Habas, P.A., Studholme, C., 2011. A Supervised Patch-Based Approach for Human Brain Labeling. Medical Imaging, IEEE Transactions on 30, 1852-1862. doi:10.1109/TMI . 2011.2156806.

Rousseau, F., Oubel, E., Pontabry, J., Schweitzer, M., Studholme, C., Koob, M., Dietemann, J.L., 2013. BTK: An Open-Source Toolkit for Fetal Brain MR Image Processing. Computer Methods and Programs in Biomedicine 109, 65-73. doi:10.1016/j.cmpb.2012.08.007.

Rutherford, M., Jiang, S., Allsop, J., Perkins, L., Srinivasan, L., Hayat, T., Kumar, S., Hajnal, J., 2008. MR Imaging Methods for Assessing Fetal Brain Development. Developmental neurobiology 68, 700-711. doi:10.1002/ dneu. 20614.

Segonne, F., Dale, A., Busa, E., Glessner, M., Salat, D., Hahn, H., Fischl, B., 2004. A Hybrid Approach to the Skull Stripping Problem in MRI. Neuroimage 22, 1060-1075. doi:10.1016/j . neuroimage.2004.03.032.

Shattuck, D.W., Sandor-Leahy, S.R., Schaper, K.A., Rottenberg, D.A., Leahy, R.M., 2001. Magnetic Resonance Image Tissue Classification Using a Partial Volume Model. NeuroImage 13, 856-876. doi:10.1006/nimg. 2000. 0730.

Smith, S.M., 2002. Fast Robust Automated Brain Extraction. Human Brain Mapping 17, 143-155. doi:10.1002/hbm. 10062.

Snijders, R., Nicolaides, K., 2003. Fetal Biometry at 14-40 Weeks' Gestation. Ultrasound in Obstetrics \& Gynecology 4, 34-48. doi:10.1046/j . 1469-0705.1994.04010034.x.

Studholme, C., Rousseau, F., 2013. Quantifying and Modelling Tissue Maturation in the Living Human Fetal Brain. International Journal of Developmental Neuroscience 32, 3-10. doi:10.1016/j . i jdevneu. 2013.06.006.

Taleb, Y., Schweitzer, M., Studholme, C., Koob, M., Dietemann, J.L. Rousseau, F., et al., 2013. Automatic Template-based Brain Extraction in Fetal MR Images. HAL Abstract 2890.

Van Leemput, K., Maes, F., Vandermeulen, D., Suetens, P., 1999. Automated Model-based Tissue Classification of MR Images of the Brain. Medical Imaging, IEEE Transactions on 18, 897-908. doi:10.1109/42.811270.

Wang, Z., Wolz, R., Tong, T., Rueckert, D., 2013. Spatially Aware Patch-Based Segmentation (SAPS): An Alternative Patch-Based Segmentation Framework, in: Menze, B., Langs, G., Lu, L., Montillo, A., Tu, Z., Criminisi, A. (Eds.), Medical Computer Vision. Recognition Techniques and Applications in Medical Imaging. Springer Berlin Heidelberg. volume 7766 of LNCS, pp. 93-103. doi:10.1007/978-3-642-36620-8_10.

Warfield, S., Kaus, M., Jolesz, F., Kikinis, R., 1998. Adaptive Template Moderated Spatially Varying Statistical Classification, in: Wells, W., Colchester, A., Delp, S. (Eds.), MICCAI'98. Springer Berlin Heidelberg. volume 1496 of $L N C S$, pp. 431-438. doi:10.1007/BFb0056228.

Wright, R., Vatansever, D., Kyriakopoulou, V., Ledig, C., Wolz, R., Serag, A., Rueckert, D., Rutherford, M.A., Hajnal, J.V., Aljabar, P., 2012. Age Dependent Fetal MR Segmentation Using Manual and Automated Approaches, in: MICCAI Workshop on Paediatric and Perinatal Imaging, Springer. pp. 97104.

Wu, Z., Ke, Q., Isard, M., Sun, J., 2009. Bundling Features for Large Scale Partial-Duplicate Web Image Search, in: CVPR'09, IEEE. pp. 25-32. 